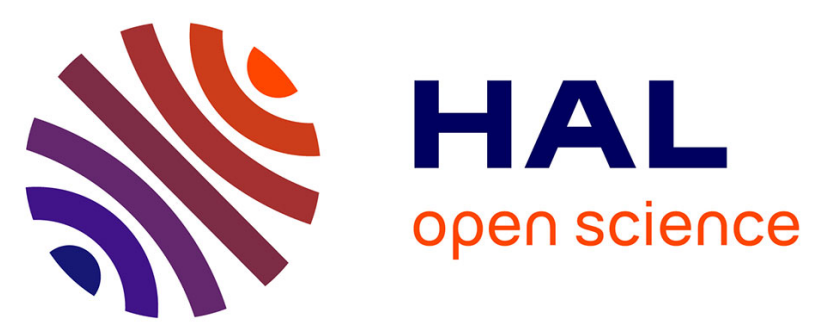

\title{
Interparticle Coulombic Decay in coupled quantum dots: enhanced energy transfer via bridge assisted mechanisms
}

Hicham Agueny, Maxime Pesche, Bastien Lutet-Toti, Tsveta Miteva, Axel

Molle, Jérémie Caillat, Nicolas Sisourat

\section{- To cite this version:}

Hicham Agueny, Maxime Pesche, Bastien Lutet-Toti, Tsveta Miteva, Axel Molle, et al.. Interparticle Coulombic Decay in coupled quantum dots: enhanced energy transfer via bridge assisted mechanisms. Physical Review B: Condensed Matter (1978-1997), 2020, 101 (19), 10.1103/PhysRevB.101.195431. hal-02877212

\section{HAL Id: hal-02877212 \\ https://hal.sorbonne-universite.fr/hal-02877212}

Submitted on 22 Jun 2020

HAL is a multi-disciplinary open access archive for the deposit and dissemination of scientific research documents, whether they are published or not. The documents may come from teaching and research institutions in France or abroad, or from public or private research centers.
L'archive ouverte pluridisciplinaire HAL, est destinée au dépôt et à la diffusion de documents scientifiques de niveau recherche, publiés ou non, émanant des établissements d'enseignement et de recherche français ou étrangers, des laboratoires publics ou privés. 


\title{
Interparticle Coulombic Decay in coupled quantum dots: enhanced energy transfer via bridge assisted mechanisms
}

\author{
Hicham Agueny, ${ }^{1}$ Maxime Pesche, ${ }^{2}$ Bastien Lutet-Toti, ${ }^{2}$ Tsveta \\ Miteva, ${ }^{2}$ Axel Molle, ${ }^{2}$ Jérémie Caillat, ${ }^{2}$ and Nicolas Sisourat ${ }^{2}$ \\ ${ }^{1}$ Department of Physics and Technology, Allegt. 55, University of Bergen, N-5007 Bergen, Norway \\ ${ }^{2}$ Sorbonne Université, CNRS, Laboratoire de Chimie Physique Matière et Rayonnement, UMR 7614, F-75005 Paris, France
}

(Dated:)

Interparticle Coulombic Decay (ICD) is an efficient energy transfer process between two weakly interacting systems. ICD was recently proposed as the underlying fundamental mechanism for technological purposes based on quantum dot nanostructures, such as wavelength-sensitive detectors. Via ICD, an excited donor quantum dot releases its excess energy by ionizing a neighbouring acceptor dot. Here, we demonstrate that the presence of a third (ICD inactive) quantum dot can serve as a bridge between the two dots, which is shown to result in an enhancement of the efficiency of the ICD-mediated energy transfer. Furthermore, our results show that this enhancement is found to be robust against the change of the characteristics of the bridge quantum dot and particularly the depth and size. On the other hand, its relative position with respect to the donor and acceptor dots is found to foster the ICD when it is located in between the two dots. Our findings provide new insights for the development of ICD-based nanostructure technologies and particularly for rational design of three coupled quantum dots. 


\section{INTRODUCTION}

Interparticle Coulombic Decay (ICD) is an efficient energy transfer process between two weakly interacting systems, first predicted theoretically in [1] and demonstrated experimentally in [2, 3]. Via ICD, an excited donor partner releases its excess energy by ionizing a neighbouring acceptor species. ICD was demonstrated to be a general phenomenon since the donor and acceptor partners may be ions, atoms, molecules [4 6], quantum wells [7 9] or quantum dots [10] 13. (see also [14]). It was also shown that the energy transfer is not restricted to the electronic degrees of freedom, and may for example involve vibronic couplings when the donor is a vibrationnaly excited molecule [15.

In terms of applications, it was recently proposed to use ICD in quantum wells and quantum dots nanostructures as a fundamental mechanism for technological purposes such as wavelength-sensitive detectors [7, 8, 12. However, to achieve a high degree of efficiency of such devices, ICD must be the dominant process. In this context, electronphonon couplings are the main competing phenomena [16. In general, the ICD efficiency decreases with increasing the distance between the donor and acceptor partners. In contrast, at a large interparticle distance, electron-phonon couplings are expected to weakly depend on this distance since the nanostructures do not strongly interact with each other [16]. It is therefore essential to determine the optimal interparticle distances that foster the ICD [10], and thus permit the enhancement of its efficiency.

In this context, the effects of the shape of quantum dots on the ICD efficiency were investigated, as well as the shape and characteristics of the involved artificial atoms [12, 17. It was also recently shown that another ICD mechanism, so-called superexchange ICD, is possible when the donor and acceptor systems are separated by a bridge atom. On the benchmark neon-helium-neon trimer, it was demonstrated that the ICD between the two neon atoms is substantially enhanced in the presence of a bridge helium atom compared to the isolated neon dimer case 18, 19. In these studies, it was shown that the energy transfer is mediated by virtual states where one electron from one of the neon is transferred to the bridge helium atom.

In the present work, we study numerically the ICD process in coupled quantum dots in order to achieve a high degree of efficiency of the ICD-mediated energy transfer between a donor and an acceptor quantum dots. In particular, we explore the possibility of controlling the ICD by changing the characteristics of the nanostructure. This is achieved using low dimensional models that effectively model coupled quantum dots, as in 10 13. The essence of our results is that a clear enhancement of the ICD rate is observed when inserting a third quantum dot. With the use of a simple physical model based on perturbation theory, we identify the origin of this enhancement and relate it to two physical mechanisms: i) the bridge dot modifies the continuum states around the resonance and ii) it yields additional virtual transitions, which occur via the superexchange ICD mechanism. Moreover, the enhancement is found to exhibit a weak dependence on the parameters of the third quantum dots (i.e. depth and size), which is an interesting finding for rational design of three coupled quantum dots. We further discuss the sensitivity of the ICD to the location of the third quantum dot with respect to the donor and acceptor dots.

Our investigation of the ICD in nanostructures focuses on semiconductors for two and three coupled quantum dots using a one-dimensional (1D) model. Although a 1D-model has obvious limitations, our main goal is, however, to search for optimal conditions, under which the ICD rate gets enhanced, which is relevant for a full dimensional model.

Our model as well as its numerical implementation based on the time-dependent Schrödinguer equation (TDSE) and perturbation theory are presented in section II. In section III we report on the ICD efficiency in the coupled quantum dots systems with respect to i) the distance between the donor and acceptor species and ii) the characteristics of the bridge quantum dot. The article ends with the conclusions of this work. Atomic units are used throughout unless stated otherwise.

\section{THEORY AND COMPUTATIONAL DETAILS}

\section{A. Quantum dots models}

We consider one-dimensional models which have proved to properly account for the essential properties of realistic three-dimensional quantum dots with respect to ICD [13. Such models provide a correct description of the electron dynamics involved in the quantum dots with less computational efforts. The effective two-electron Hamiltonian in our systems is given as

$$
H\left(z_{1}, z_{2}\right)=h_{1}\left(z_{1}\right)+h_{2}\left(z_{2}\right)+V_{C}\left(z_{1}, z_{2}\right)
$$

where $z_{i}(i=1,2)$ denotes the coordinate of the $i$ th electron. The one-electron Hamiltonian $h_{i}$ is of the form

$$
h_{i}\left(z_{i}\right)=-\frac{1}{2} \frac{\partial^{2}}{\partial z_{i}^{2}}+V_{Q D}\left(z_{i}\right),
$$



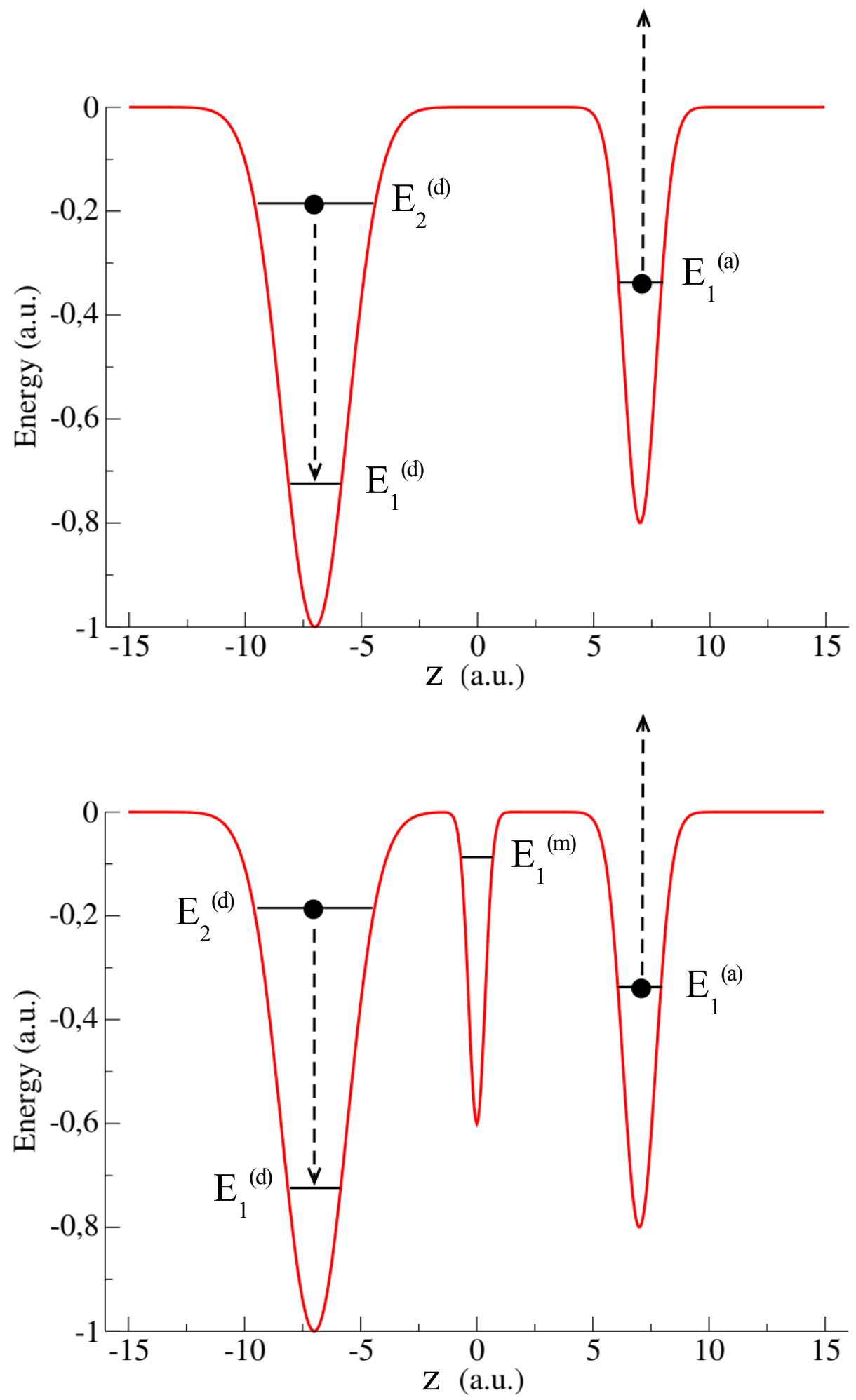

FIG. 1. Scheme of the ICD in two (upper panel) and three (lower panel) coupled quantum dots. Via ICD, the left (donor) quantum dot releases its excess energy by ionizing the right (acceptor) dot, as indicated by the arrows. The quantum dots are represented by effective one-dimensional potentials as in [10 13. $E_{n}^{(j)}$ denote the one-electron energies (see text). 
where $V_{Q D}\left(z_{i}\right)$ is the electron-dot potential (as shown in Fig. 1), built on Gaussian potentials as in [10]:

$$
V_{Q D}\left(z_{i}\right)=-Z_{d} e^{-\sigma_{d}\left(z_{i}+R / 2\right)^{2}}-Z_{m} e^{-\sigma_{m}\left(z_{i}-b_{m}\right)^{2}}-Z_{a} e^{-\sigma_{a}\left(z_{i}-R / 2\right)^{2}} .
$$

Here, $Z_{j}$ and $\sigma_{j}(j=d, m, a)$ are respectively the depth and size of the donor, mediator and acceptor quantum dot potentials. $R$ is the distance between the donor and acceptor quantum dots, which are located symmetrically around the origin, and $b_{m}$ is the coordinate of the mediator center. In this framework, a two coupled quantum dots is modeled by setting $Z_{m}=0$. In Eq. 1. the single-particle Hamiltonians $h_{1}$ and $h_{2}$ are coupled via the interaction term $V_{C}\left(z_{1}, z_{2}\right)$. The latter is a repulsive soft Coulomb potential and is expressed as

$$
V_{C}\left(z_{1}, z_{2}\right)=\frac{1}{\sqrt{\left(z_{1}-z_{2}\right)^{2}+\alpha \exp \left[-\beta\left(z_{1}-z_{2}\right)^{2}\right]}} .
$$

This model potential has been chosen to avoid the singularity at $z_{1}=z_{2}$. The same model has been used in previous works to describe the correlated electron-electron interaction in connection with the ICD in two coupled quantum dots (see e.g. [10]).

\section{B. Dynamics and decay widths}

The electron dynamics of the nanostructures modeled as above is governed by the TDSE

$$
\left[H\left(z_{1}, z_{2}\right)-i \frac{\partial}{\partial t}\right] \psi\left(z_{1}, z_{2}, t\right)=0 .
$$

We construct the initial wave function from the eigenstates of the one-electron Hamiltonian, in which one electron is localized in the excited state $\phi_{2}^{(d)}$ of the donor quantum dot and the other in the ground state $\phi_{1}^{(a)}$ of the acceptor quantum dot:

$$
\psi\left(z_{1}, z_{2}, t=0\right)=\frac{1}{\sqrt{2}}\left[\phi_{2}^{(d)}\left(z_{1}\right) \phi_{1}^{(a)}\left(z_{2}\right)+\phi_{2}^{(d)}\left(z_{2}\right) \phi_{1}^{(a)}\left(z_{1}\right)\right] .
$$

The energy $(E)$ of the initial state is above the lowest ionization threshold $\left(E_{\infty}\right)$ of the two-electron system (i.e. socalled resonant, or quasi-bound, state). The one-electron states denoted by $\phi_{n}^{(j)}(j=d, m, a)(n=1,2)$ are solutions of the time-independent Schrödinger equation

$$
h_{i}\left(z_{i}\right) \phi_{n}^{(j)}\left(z_{i}\right)=E_{n}^{(j)} \phi_{n}^{(j)}\left(z_{i}\right) .
$$

The initial wave-function is chosen to be symmetric with respect to electron permutation, which corresponds to a singlet spin state. We have also performed the calculations for the triplet state case. Similar conclusions as the ones reported below were reached. Note that the symmetry is conserved throughout propagation. At the end of the propagation, the decay width $\Gamma$ for a given quantum dot system is obtained by fitting the computed values of the autocorrelation function:

$$
a(t)=|\langle\psi(t=0) \mid \psi(t)\rangle|^{2}
$$

to the generic decay function $e^{-\Gamma t}$.

\section{Perturbation model}

To help the interpretation of the results stemming from the TDSE, we use a formalism that is reported in 20, 21. In our work, the formalism is simplified by making assumptions about the choice of the wave functions involved in the couplings, as shown below (see also [18] and references therein). Here, our main goal is not to obtain quantitatively accurate results for the ICD width as much as to identify and discuss the main contributions that lead to the enhancement of the ICD width in the presence of a third quantum dot. In this context, the decay width is given by

$$
\Gamma=2 \pi\left|\left\langle\psi(t=0)|\hat{H}-E| \psi_{f}\right\rangle\right|^{2}
$$


where $\psi(t=0)$ and $E$ are the initial state and its energy, respectively (see Eq. 6). The final state $\psi_{f}$ is in our model

$$
\psi_{f}=N\left[\psi_{k}+\frac{\left\langle\psi_{d m}|\hat{H}| \psi_{k}\right\rangle}{\left(E-E_{d m}\right)} \psi_{d m}\right]
$$

where

$$
\begin{gathered}
\psi_{k}=\frac{1}{\sqrt{2}}\left[\phi_{1}^{(d)}\left(z_{1}\right) k\left(z_{2}\right)+\phi_{1}^{(d)}\left(z_{2}\right) k\left(z_{1}\right)\right], \\
\psi_{d m}=\frac{1}{\sqrt{2}}\left[\phi_{2}^{(d)}\left(z_{1}\right) \phi_{1}^{(m)}\left(z_{2}\right)+\phi_{2}^{(d)}\left(z_{2}\right) \phi_{1}^{(m)}\left(z_{1}\right)\right],
\end{gathered}
$$

$N$ a normalization factor and $E_{d m}$ the energy of the latter state. In Eq. 11 . $k$ represents the continuum state at the energy of the ICD electron (i.e. $E-E_{\infty}$ ). The second term in Eq. 10 corresponds to the superexchange ICD term in which the energy transfer is mediated by the state where one electron sits in the donor dot while the second electron is in the bridge dot. Note that there are other couplings that could be included in the second term (e.g. one electron is either in the ground state of the donor or of the acceptor dots and the second electron in the bridge). Our calculations, however, show that their contributions are insignificant and thus are omitted in Eq. 10.More details about the evaluation of the decay width using Eq. (10) can be found in [18].

\section{Computational details}

We used the same parameters to model the donor and acceptor dots as in [10, i.e. $\left(Z_{d}, Z_{a}\right)=(1.0,0.8)$ a.u. and $\left(\sigma_{d}, \sigma_{a}\right)=(0.25,1.0)$ a.u., while for the mediator quantum dot a broad range of parameters is considered (see below). With the use of these parameters, the donor dot supports two-bound states and their eigenenergies are labeled $E_{1}^{(d)}$ and $E_{2}^{(d)}$, while the acceptor and mediator dots support only one-bound state each and the corresponding energies are labeled $E_{1}^{(a)}$ and $E_{1}^{(m)}$, respectively. These single electron energies are given in Fig. 1 for $\mathrm{R}=14$ a.u. and $b_{m}=0$. Note that they do not vary by more than $20 \%$ with respect to these parameters in the range of values employed in this work. The time-independent Schrödinger (see Eq.7) is solved using a Lagrange-mesh method 22, 23, with variational basis functions of a sinus form. In the interaction term $V_{C}\left(z_{1}, z_{2}\right), \alpha$ and $\beta$ are soft parameters set to 0.01 and 100 a.u., respectively [10].

The time evolution of the electronic wave function $\psi\left(z_{1}, z_{2}, t\right)$, which satisfies the TDSE (see Eq. 5), is solved numerically using a split-operator method combined with a fast Fourier transform algorithm as in [24]. This is carried out on a symmetric $2 \mathrm{D}$ grid of size $L=127.75$ a.u. with the grid spacing $\delta z=0.25$ a.u., i.e. 512 grid points along each direction. The time step used in the calculation is $\delta t=0.09$ a.u. The convergence was checked by performing additional calculations with twice the size of the box and a smaller time step. Furthermore, a complex absorbing potential (CAP) placed at positions $\pm z_{C A P}$ along each coordinate is employed to avoid artificial reflections. It is expressed as [11]

$$
W_{C A P}=-i \eta\left|z_{i} \pm z_{C A P}\right|^{k} \Theta\left(z \pm z_{C A P}\right),
$$

where $\eta$ and $k$ are the strength and the order of the CAP, respectively. $\Theta$ is the Heavyside step function. In these calculations, we used $\eta=0.003$ and $k=2$. The boundary is chosen such that $z_{C A P}= \pm 0.85 L$. Convergence of the results with respect to the CAP parameters and grid size have been checked.

We applied the fitting procedure to obtain the decay widths (see Eq. 8) at different final times, ranging from 6200 to 12400 a.u.. The results do not change significantly with respect to the latter values.

\section{RESULTS AND DISCUSSION}

The ICD mechanisms to be discussed are schematically depicted in Fig. 1 for the case of two (upper panel) and three coupled quantum dots (lower panel). The top diagram represents a direct process, in which the excess energy of the donor can be used to directly ionize the electron from the acceptor. In the case of three coupled quantum dots, the excess energy is mediated by the presence of a third quantum dot located between the donor and acceptor during 


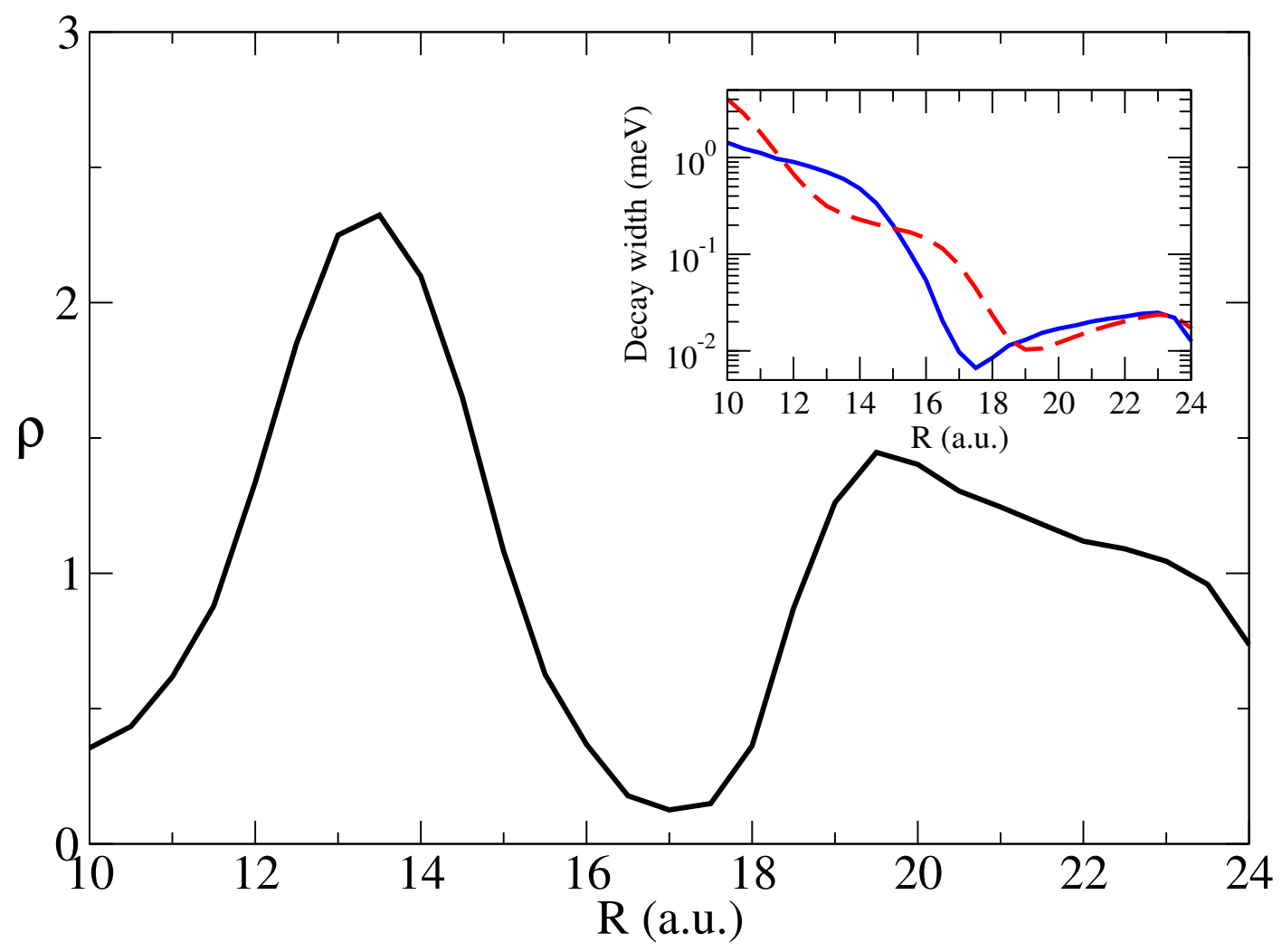

FIG. 2. Ratio between the decay widths for three and two coupled quantum dots as a function of the distance between the donor and acceptor dots (R). Inset: Decay widths for two (dashed red line) and three (blue line) coupled quantum dots. The parameters (see text) of the bridge quantum dot are $\left(Z_{m}=0.6 ; \sigma_{m}=4.0\right)$.

the ICD. In the perturbation theory picture, this additional effect is taken into account via an extra term related to the superexchange ICD, as outlined in connection with Eq. 10. This mechanism has been discussed for trimers and has been shown to lead to an enhancement of the efficiency of the ICD width [18].

Stimulated by these recent findings [18] and by fundamental interest of quantum dots for nanotechnological purposes, we aim in this work at searching for optimal conditions characterizing a third quantum dot, and under which the ICD width gets enhanced. Here the characteristics of this third quantum dot (depth, size and relative position), which is referred to as bridge, are chosen such that the energies of the bound states of the donor and acceptor remain unchanged with respect to the case of two coupled quantum dots. This is an important condition for ensuring the physical interpretation of the efficiency of the ICD in the presence of a bridge quantum dot.

We consider both scenarios depicted in Fig. 1 and calculate the corresponding decay widths. To evaluate the enhancement of the ICD, we present in Fig. 2 the ratio

$$
\rho=\frac{\Gamma_{3 Q D}}{\Gamma_{2 Q D}}
$$

between the decay widths for the three coupled $\left(\Gamma_{3 Q D}\right)$ and two coupled $\left(\Gamma_{2 Q D}\right)$ dots as a function of the distance $R$ between the donor and acceptor dots (all other parameters fixed) is shown in Fig. 2. The widths for each system are displayed in the inset of the same figure. The parameters of the bridge quantum dot are $Z_{m}=0.6, \sigma_{m}=4.0$ and $b_{m}=0.0$ (i.e. the bridge dot is at the midpoint between the donor and acceptor dots). It is shown that, in general, the decay widths decrease with increasing $R$ for both systems. However, this trend is not monotonous as already discussed in 10]: for some interparticule distances the electron-electron interaction creates an effective potential that may trap the ICD electron, delaying the decay process (e.g at $\mathrm{R} \simeq 17$ and 19 a.u. for the two and three QD cases, respectively). The ratio between the decay widths varies between about 0.1 and 2.3 , showing that a significantly larger ICD efficiency (i.e. a faster decay) can be achieved for some interparticle distances in the presence of the bridge dot. As seen in the figure, the largest enhancement of the decay process is obtained here at about $R \simeq 13.5$ a.u.

To provide insights into the observed enhancement, we make use of a simple physical model as described in Section IIC The model is based on perturbation theory and has the advantage of separating direct processes from indirect 


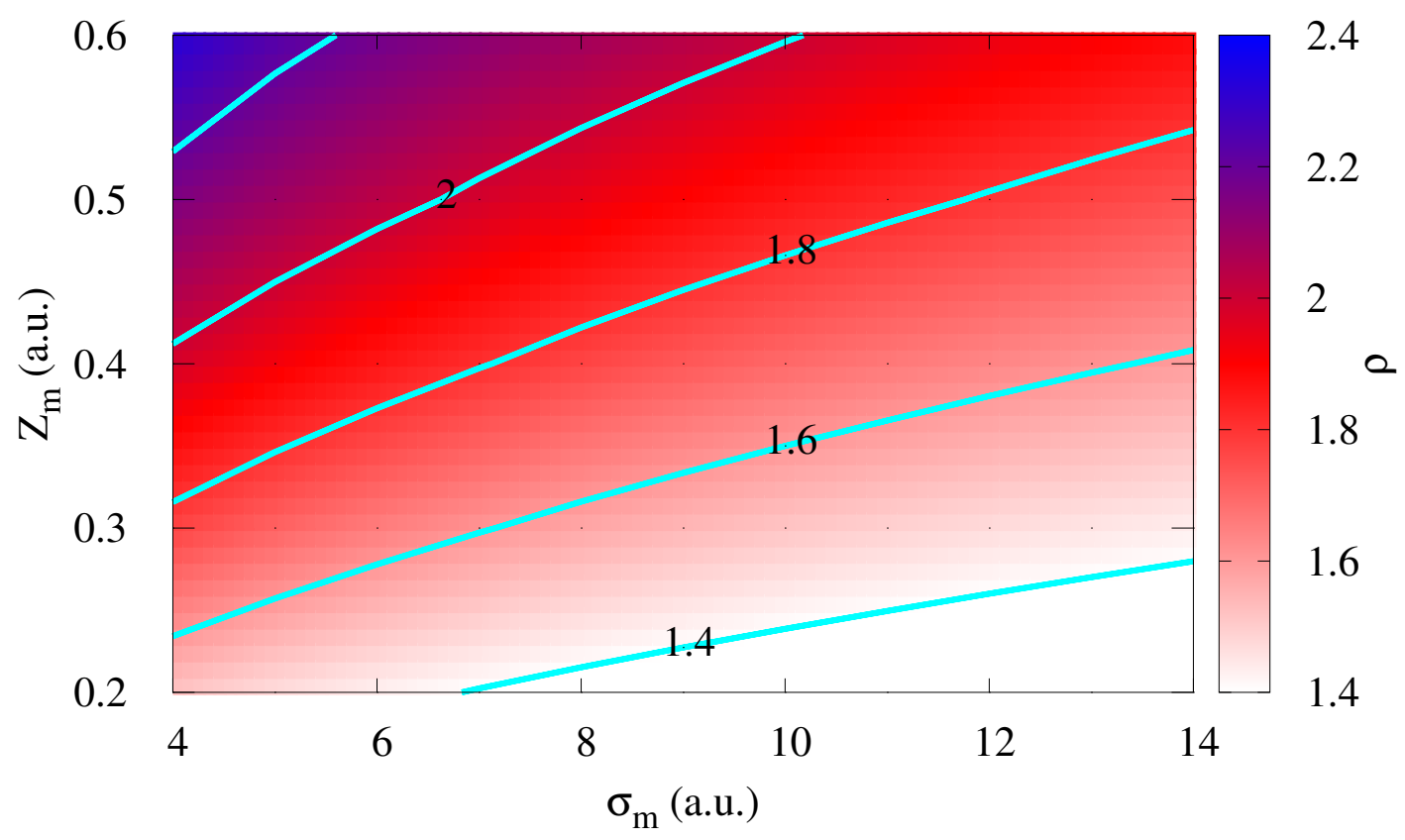

FIG. 3. Ratio between the decay widths for three and two coupled quantum dots as a function of the parameters (see text) of the bridge quantum dot, for $R=13.5$ a.u. Some contour lines are indicated by the cyan lines.

ones. The model, therefore enables us to identify the main contributions responsible of the observed enhancement of the ICD width in Fig. 2. Here, the bride quantum dot can lead to the observed enhancement around $R \simeq 13.5$ a.u. via two mechanisms: it can modify the continuum states around the resonance (see 7 for more details) and can participate in the energy transfer via superexchange ICD. To disentangle and quantify both contributions we have computed the decay widths using the perturbation model reported in IIC. Above $R=12$ a.u., the results stemming from this model agrees quantitatively with that of the numerically exact TDSE calculations. Below $R=12$ a.u., the coupling between $\psi_{k}$ and $\psi_{d m}$ becomes too strong and the perturbation correction is not adequate. We, therefore, focus on the results of the model within its range of validity (i.e. $R>12$ a.u.). By removing the superexchange ICD term in Eq. 10 (i.e. $\psi_{f}=\psi_{k}$ ), it is possible to quantify the contributions of both mechanisms discussed above. These calculations show that both mechanisms contribute nearly equally for $R \sim 13.0-18.0$ a.u. (not shown). At larger distances, the superexchange ICD mechanism does not contribute since the coupling between $\psi_{d m}$ and $\psi_{k}$ decreases exponentially with the distance (see [18). We mention here that the perturbation model predicts that the superexchange ICD mechanism largely dominates at distances below 12.0-13.0 a.u. However, as noted above the model is not quantitative in this interparticle distance range and further works are needed.

We now investigate the sensitivity of ICD with respect to the parameters of the bridge species. In Fig. 3 , we present the ratio $\rho$ (Eq. 14) as a function of $Z_{m}$ and $\sigma_{m}$. The distance between the donor and acceptor species is fixed at $R=13.5$ a.u. and $b_{m}$ is kept equal to 0 (i.e. the bridge dot is again fixed at the midpoint between the donor and acceptor dots). Our results show that, for $Z_{m}$ ranging from 0.3 to 0.6 and $\sigma_{m}$ ranging from 4 to 10 a.u, the ICD width is enhanced in the presence of the bridge dot by a factor comprised between about 1.6 and 2.4.

Furthermore, in Fig. 4 we show the decay width as a function of the position $b_{m}$ of the bridge quantum dot, for $R=13.5$ a.u. We report only results for values of $b_{m}$ which do not modify significantly the energies of the donor and acceptor quantum dots, as compared to the centered case $\left(b_{m}=0\right)$. One can see in the middle panel that the enhancement of the ICD process is maximal when the bridge dot is at midpoint between the two other quantum dots. The ICD process becomes weaker as the bridge dot approaches the acceptor one (i.e. $b_{m}>0$ ). The energies of the donor are significantly modified when the bridge dot is moved towards it (i.e. $b_{m}<0$ ). A change in the donor potential affects the efficiency of the ICD process. As mentioned above, we are interested in the enhancement of the ICD process owing only to the presence of the third dot. We therefore do not report results for $-13<b_{m}<0$.

The left and right panels in Fig. 4 show the decay width when the bridge dot is located on the left side of the donor 


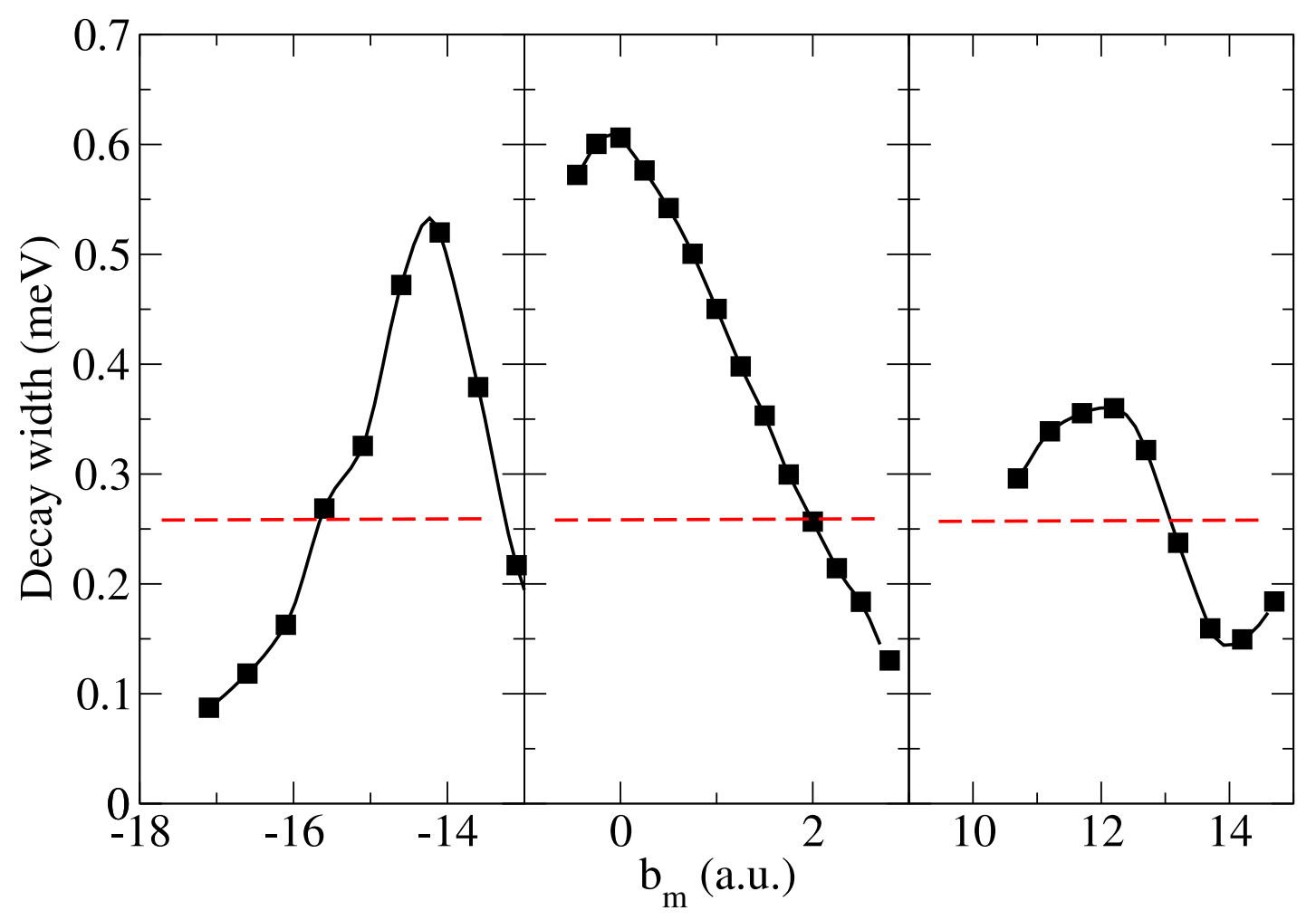

FIG. 4. Decay widths as a function of the position of the bridge quantum dot, for $R=13.5$ a.u. (i.e. the donor and acceptor dots are fixed at -6.75 a.u. and 6.75 a.u., respectively.). The red dashed-line indicate the decay width in absence of the bridge. Note that we have limited the range of $b_{m}$ to the values that do not change significantly the energies of the donor and acceptor dots with respect to the two coupled quantum dots case.

$\left(b_{m}<-R / 2\right)$ or on the right side of the acceptor $\left(b_{m}>+R / 2\right)$, respectively. It is interesting to note that the ICD is substantially enhanced when the bridge dot is placed in the left side of the donor dot, showing that the relative position of the bridge, donor and acceptor dots is also a relevant parameter for the optimization of the ICD rate. The observed behavior of the decay widths, reported in Fig. 4 is related to interferences between the direct path (i.e. $\left.\psi(t=0) \rightarrow \psi_{k}\right)$ and the one corresponding to the transition via the virtual state $\psi_{d m}$ (i.e. via the superexchange ICD mechanism). These interferences lead to the enhancement or suppression of the decay width depending on the location of the bridge quantum dot. This can be understood from the perturbation model defined above and which incorporates virtual transitions in the final state, as shown in Eq. 10p. A similar effect has been recently discussed for three body ICD processes occurring in atomic clusters 25.

At this point, we conclude that the efficiency of the ICD-mediated energy transfer can be achieved for a broad range of parameters characterizing the inserted bridge quantum dot. This is an interesting finding for rational design of three coupled quantum dots.

\section{CONCLUSION}

We have shown that energy transfer mediated by the Interparticle Coulombic Decay (ICD) process between two quantum dots can be substantially enhanced in the presence of a third bridge dot. The efficiency of the process can thus be improved by more than a factor of 2 . Furthermore, we have investigated the mechanisms leading to such increase and how the latter depends on the characteristics (depth, size and relative position) of the bridge species. It was found that the efficiency of the ICD is robust against the change of the depth and size of the inserting bridge dot. On the other hand, this efficiency was shown to be sensitive to the relative position of the bridge dot with respect to the donor and acceptor quantum dots. Our findings, therefore, offer alternative routes to manipulate the ICD and ultimately design three coupled quantum dots. This work provides new insights into the energy transfer processes in nanostructures and should be useful for the development of quantum dot based technologies. 


\section{ACKNOWLEDGEMENT}

This project has received funding from the LabEx MiChem part of French state funds managed by the ANR within the 'Investissements d'Avenir' program under reference ANR-11-IDEX-0004-02. The computations were performed on resources provided by UNINETT Sigma2 - the National Infrastructure for High Performance Computing and Data Storage in Norway.

[1] L. S. Cederbaum, J. Zobeley, and F. Tarantelli, Phys. Rev. Lett. 79, 4778 (1997).

[2] S. Marburger, O. Kugeler, U. Hergenhahn, and T. Möller, Phys. Rev. Lett. 90, 203401 (2003).

[3] T. Jahnke, A. Czasch, M. S. Schöffler, S. Schössler, A. Knapp, M. Käsz, J. Titze, C. Wimmer, K. Kreidi, R. E. Grisenti, A. Staudte, O. Jagutzki, U. Hergenhahn, H. Schmidt-Böcking, and R. Dörner, Phys. Rev. Lett. 93, 163401 (2004).

[4] V. Averbukh, P. V. Demekhin, P. Kolorenč, S. Scheit, S. D. Stoychev, A. I. Kuleff, Y.-C. Chiang, K. Gokhberg, S. Kopelke, N. Sisourat, and L. S. Cederbaum, J. Electron Spectrosc. Relat. Phenom. 183, 36 (2011).

[5] U. Hergenhahn, J. Electron Spectrosc. Relat. Phenom. 184, 78 (2011).

[6] T. Jahnke, J. Phys. B: At. Mol. Opt. Phys. 48, 082001 (2015)

[7] T. Goldzak, L. Gantz, I. Gilary, G. Bahir, and N. Moiseyev, Phys. Rev. B 91, 165312 (2015)

[8] T. Goldzak, L. Gantz, I. Gilary, G. Bahir, and N. Moiseyev, Phys. Rev. B 93, 045310 (2016)

[9] T. Goldzak, Mol. Phys. 117, 2179 (2019).

[10] A. Bande, K. Gokhberg, and L. S. Cederbaum, J. Chem. Phys. 135, 144112 (2011)

[11] F. M. Pont, A. Bande, and L. S. Cederbaum, Phys. Rev. B 88, 241304 (2013)

[12] P. Dolbundalchok, D. Pelez, E. F. Aziz, and A. Bande, J. Comp. Chem. 37, 2249 (2016).

[13] F. M. Pont, A. Bande, and L. S. Cederbaum, J. Phys: Condens. Matter 28, 075301 (2016)

[14] See http://www.pci.uni-heidelberg.de/tc/usr/icd/ICD.refbase.html for the complete list of ICD papers.

[15] L. S. Cederbaum, Phys. Rev. Lett. 121, 223001 (2018)

[16] A. Bande, Mol. Phys. 117, 2014 (2019)

[17] F. Weber, E. F. Aziz, and A. Bande, J. Comput. Chem. 38, 2141 (2017)

[18] T. Miteva, S. Kazandjian, P. Kolorenč, P. Votavová, and N. Sisourat, Phys. Rev. Lett. 119, 083403 (2017).

[19] P. Votavová, T. Miteva, S. Engin, S. Kazandjian, P. c. v. Kolorenč, and N. Sisourat, Phys. Rev. A 100, 022706 (2019)

[20] U. Fano, Phys. Rev. 124, 1866 (1961).

[21] G. Howat, T. Åberg, and O. Goscinski, J. Phys. B At. Mol. Opt. Phys. 11, 1575 (1978).

[22] D. Baye and P. H. Heenen, Journal of Physics A: Mathematical and General 19, 2041 (1986)

[23] H. Agueny, A. Makhoute, A. Dubois, and J. P. Hansen, Phys. Rev. A 93, 012713 (2016)

[24] H. Agueny and J. P. Hansen, Phys. Rev. A 98, 023414 (2018)

[25] R. Bennett, P. Votavová, P. Kolorenč, T. Miteva, N. Sisourat, and S. Y. Buhmann, Phys. Rev. Lett. 122, 153401 (2019) 